\title{
Errata: Influence of loupes and age on the near visual acuity of practicing dentists
}

\author{
Martina Eichenberger, ${ }^{a}$ Philippe Perrin, ${ }^{a}$ Klaus W. Neuhaus, ${ }^{a}$ Ueli Bringolf, ${ }^{b}$ and Adrian Lussia ${ }^{a}$ \\ a University of Bern, School of Dental Medicine, Department of Preventive, Restorative and Pediatric Dentistry, \\ $\mathrm{CH}-3010$ Bern, Switzerland \\ b Nordstrasse 26, CH-8200 Schaffhausen, Switzerland
}

[DOI: $10.1117 / 1.3600762]$

This article [J. Biomed. Opt. 16, 035003 (2011)] was originally published online on 16 Mar 2011 with an error in Table 4: the columns titled " $95 \% \mathrm{Cl}$ " were reversed. The corrected table appears below.

The article was corrected online on 27 May 2011.

Table 4 Visual acuities for the groups with a natural visual acuity $\geq 1.1$ and $<1.1$ under different magnification conditions: natural visual acuity (NV), single lens loupe (S), Galilean system (G), and Keplerian system $(\mathrm{K})$. Within the columns, significant differences are represented by the different superscript letteres (a: $p<0.001, \mathrm{~b}$ : $\mathrm{p}<0.05$ ).

\begin{tabular}{|c|c|c|c|c|c|c|}
\hline \multirow[b]{2}{*}{ Condition } & \multicolumn{3}{|c|}{ Visual acuity $\geq 1.1$} & \multicolumn{3}{|c|}{ Visual acuity $<1.1$} \\
\hline & Mean & Median & $95 \% \mathrm{Cl}$ & Mean & Median & $95 \% \mathrm{Cl}$ \\
\hline$N V^{a}$ & 1.33 & 1.36 & $(1.28 ; 1.39)$ & 0.97 & 1.01 & $(0.94 ; 1.01)$ \\
\hline$S, 2 x^{b}$ & 1.58 & 1.31 & $(1.31 ; 1.56)$ & 1.19 & 1.31 & $(1.13 ; 1.31)$ \\
\hline$G, 2.5 x^{b}$ & 2.66 & 2.74 & $(2.37 ; 2.74)$ & 2.13 & 2.32 & $(1.99 ; 2.32)$ \\
\hline $\mathrm{K}, 4.3 \mathrm{x}^{\mathrm{b}}$ & 5.12 & 4.62 & $(4.31 ; 5.22)$ & 4.00 & 4.00 & $(3.46 ; 4.31)$ \\
\hline
\end{tabular}

